\title{
THE STRUCTURAL FEATURES OF EPILOIA, WITH SPECIAL REFERENCE TO ENDOCARDIAL FIBROELASTOSIS
}

\author{
BY \\ L. CROME \\ From the Department of Neuropathology, the Fountain Hospital, Tooting, London
}

(RECEIVED FOR PUBLICATION OCTOBFR 20, 1953)

While the morbid anatomical features of epiloia are probably its best-known aspect, the condition as a whole is obscure. It is rare, and general pathologists have few opportunities to study it. The object of this communication is to enumerate the lesions of epiloia, and to add another one, fibroelastosis of the heart, to the rather long list of structural abnormalities encountered in this condition.

The material used in the preparation of this paper included four cases at the Fountain Hospital, and, thanks to Dr. J. G. Greenfield and Dr. W. H. McMeneney, I had also access to the collection at the National and the Maida Vale hospitals, and in addition histological sections of cases were lent to me by Dr. R. M. Norman, Dr. W. Thomas Smith, and Dr. A. J. N. Warrack.

The characteristic case of epiloia (Table I) has tuberous sclerosis of the brain manifested by epilepsy with or without mental defect, adenoma sebaceum of the face, and one or more of the abnormalities listed in this table. Adenoma sebaceum may, however, be absent, especially in infancy and early childhood, and incomplete, formes frustes of the disease, such as adenoma sebaceum without neurological symptoms, or cases with only visceral lesions, have been described, particularly among the patients' relatives and in the late, adult type of the disease. It should be noted in this connexion that less extensive cerebral lesions need not necessarily give rise to either epilepsy or mental defect, and may easily remain undetected in post-mortem examinations.

The characteristic hard nodules of tuberous sclerosis can be palpated better than seen in the freshly removed brain, but may become more conspicuous in the fixed material after the meninges have been stripped. They are situated in the cerebral hemispheres, vary greatly in number and size, and frequently calcify. Lesions have also been described in the cerebellum, brain-stem, and spinal cord ; these are, however, relatively uncommon, The larger nodules are well seen in sections stained by the Nissl method as areas of absent or deficient cortical staining. Corresponding sections stained by any of the glial methods show as areas of dense fibrous gliosis situated mainly in the cortex, but also in the subjacent white matter.

Cortical lamination in the nodules is absent, the number of nerve cells greatly diminished, the remainder being pyknotic, shrunken, sometimes abnormally large and orientated at unusual angles. The number of glial cells, chiefly of astrocyte type, is, on the other hand, increased. Some of them may also be abnormally large, having several nuclei and homogeneous cytoplasm. These are particularly frequent in the gliotic nodules of the white matter and in the more superficial portions of the cortical nodules. The bulk of the substance of the nodules is formed, however, by a dense feltwork of glial fibres. The distribution of the fibrous gliosis is quite uneven and dense patches or tufts of glial fibres are frequently seen upon the background of only moderately gliosed tissue.

"Candle-guttering" is the name given to subependymal nodules projecting into the ventricles-usually the lateral ventricles. They tend to be more cellular than the cortical nodules and it is noteworthy that such frank gliomataastrocytoma or spongioblastoma-as have been described in epiloia, were also situated near the lateral ventricles, and were therefore probably derived from the nodules of "candle-guttering."

The list of skin lesions in epiloia is, for obvious reasons, longer than that in other organs. The commonest amongst them is adenoma sebaceum, an acneiform, papular, colourless, or brownish rash of the face, which may only appear in later childhood or after puberty, when it often takes on a reddish tinge. The term "adenoma" is unfortunate. The usual microscopical appearances indicate only a moderate increase and a somewhat 
TABLE I

EPILOIA

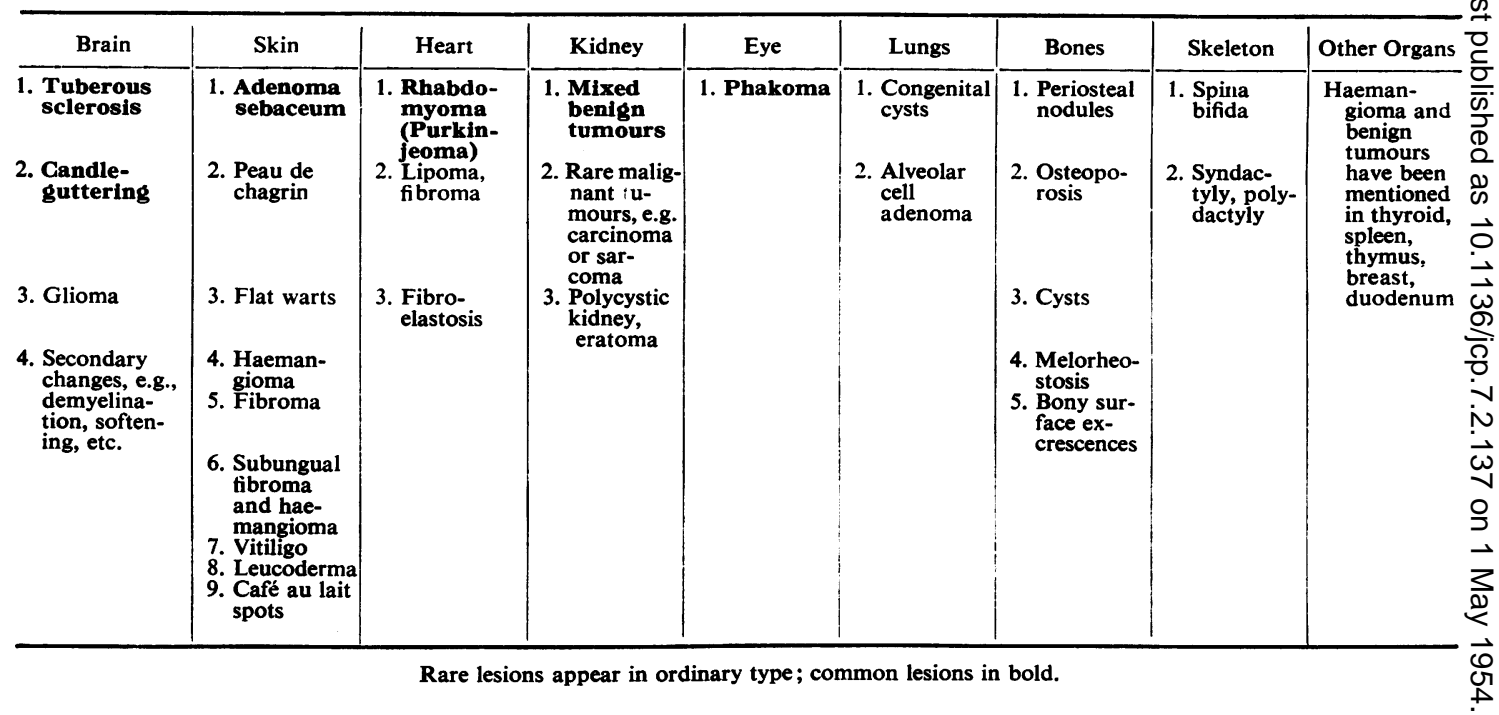

disordered arrangement of the sebaceous glands, blood vessels, and fibrous tissue in the more superficial portion of the dermis. There is also often hyperkeratosis, thinning of the epidermis, and irregularity in the length of the rete pegs.

Another characteristic skin lesion is "peau de chagrin"-areas of shagreen skin often situated on the buttocks or thighs. They are formed by closely set, flattish, or more elongated outgrowths of the superficial layer of the skin.

The cardiac lesions, "rhabdomyomata," are among the more familiar features of epiloia. They are formed by large cells the cytoplasm of which is sometimes coarsely granular, but more commonly forms radial or tangential fibrils, or bundles of fibrils, leaving much or even most of the intracellular space empty. A strip of cytoplasm in some of these cells may show distinct transverse striation, particularly if stained with Heidenhain's haematoxylin (Fig. 1). These cells are structurally identical with the normal Purkinje's cells of the heart, and their tumour-like accumulation in epiloia may be described more appropriately as "purkinjeoma" rather than the customary " rhabdomyoma." These cells are by no means always arranged in tumour-like nodules. They sometimes lie singly or in small separated groups in the subendocardial layer or within fibrotic areas in the myocardium.

Two of the cases showed congenital fibroelastosis of the heart. The left ventricle was hypertrophied (Fig. 2), some of the chordae tendineae thickened and the endocardium over the mitral aspect of the interventricular septum opaque and thick. Histological examination (Fig. 3) showed a considerable increase in fibrous and elastic tissue throughout the endo- $\approx$ cardium involving in a few places the subjacent $\stackrel{\mathbb{Q}}{\square}$ muscle (Fig. 4). Nests of Purkinje cells were $\overrightarrow{\overrightarrow{0}}$ sometimes seen within this fibroelastic layer or 3 just under it.

The kidney is one of the most frequently affected organs in epiloia. The structural changes, hamartomata or tumours, vary greatly in size, number, and histological structure. They show frequently a mixed and disorderly arrangement of such structures as fat, blood vessels, muscle, and fibrous $\frac{O}{2}$ tissue, some of which are reminiscent of elements

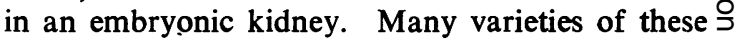
malformations and tumours have been described $\rightarrow$ as fibroma, myoma, angioma, endothelioma, lipoma, perithelioma, being present usually in N combination with each other. Malignant renal tumours-carcinomata and sarcomata-have also 0 been described. It is worth mentioning that while $\omega$ the histological appearances in some of the? tumours described in the literature were certainlyc suggestive of malignancy, no published records of $\mathbb{D}$ secondaries in other organs have been found. Professor Dorothy Russell (verbal communica- $\frac{T}{T}$ tion) has, however, seen such a case with secon- $\frac{O}{\mathbb{D}}$ daries of a lipofibrosarcoma.

Polycystic kidneys and teratomata have also $\stackrel{\mathbb{Q}}{\stackrel{2}{2}}$ been described in epiloia.

Another lesion in epiloia is the phakoma of the retina, a whitish plaque or nodule which may beg 


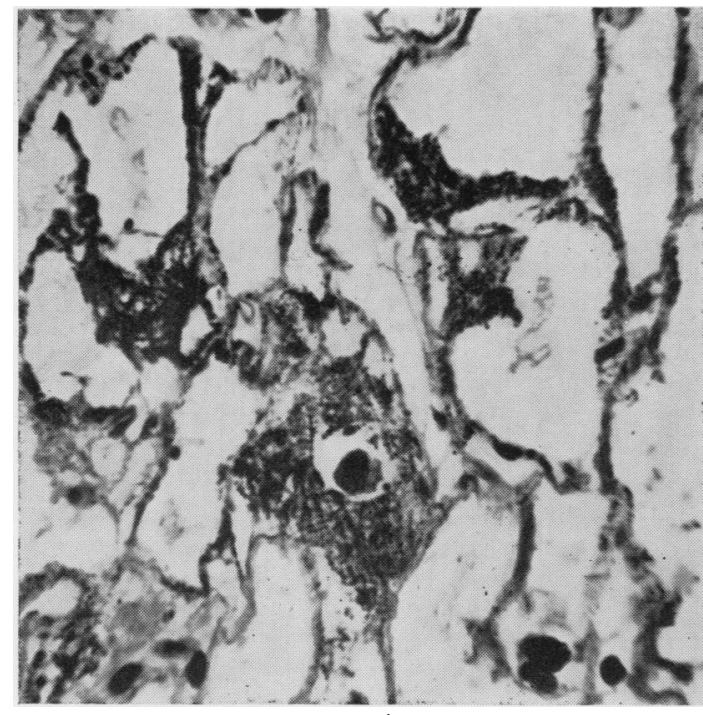

Fig. 1.

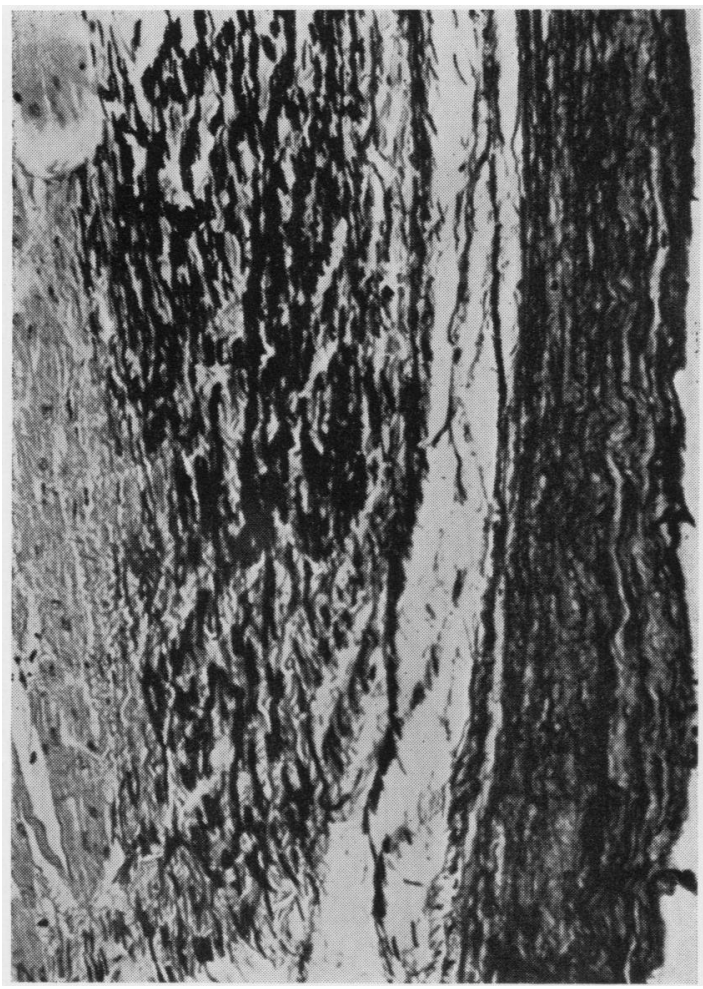

FIG. 3.

FIG. 1.-Cells in purkinjeoma showing transverse striation. Heidenhain's haematoxylin $\times 180$.

FIG. 2.-The left ventricle is hypertrophied. The endocardium and some chordae tendineae are thickened and opaque.

Fig. 3.-Numerous elastic fibres are present in the thickened endocardium. Verhoeff's elastic stain $\times 180$.

FiG. 4. - Fibroelastosis of endocardium and subjacent muscle. Verhoeff's elastic stain $\times 44$

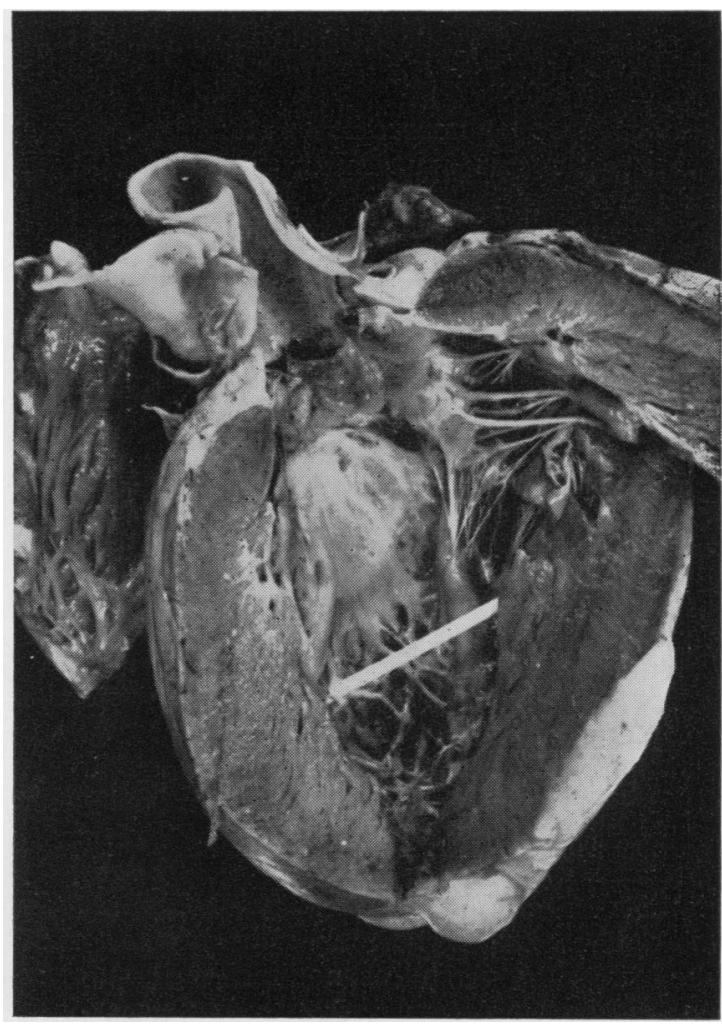

FIG. 2.

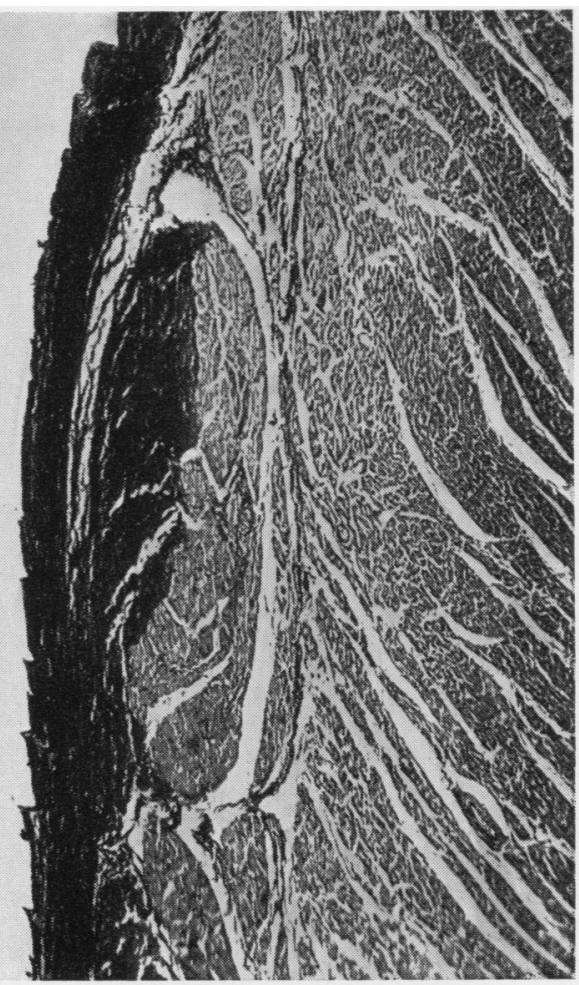

Fig. 4. 
visible on ophthalmoscopic examination. It may consist of rather uniform, densely arranged glial cells and fibres.

Many other lesions have been described in the numerous case reports of epiloia available in the literature. Some of them have only been mentioned once in single cases, and all are probably rarer than those already enumerated. Amongst them were haemangiomata, cysts, and benign uniform or mixed tumours in such organs as the thyroid, spleen, thymus, breast, and duodenum. The material used in the preparation of this communication included an example of a cystic lung and of a papillary alveolar cell adenoma of the lung.

\section{Discussion}

Congenital fibroelastosis of the endocardium has been recently discussed by several workers and the subject has been well reviewed by Gowing (1953). The condition affects infants and young children, is believed to be invariably fatal, and is characterized by progressive heart failure associated with cardiac hypertrophy and endocardial thickening. The association of endocardial fibroelastosis with epiloia has not received any attention, although it was undoubtedly present in at least two of the previously reported cases: in Case 15 of the series reported by Critchley and Earl (1932) and in the case of Norman and Taylor (1940). Two further instances of this association were, as mentioned above, found in the present material. It may well be that its incidence in epiloia, the frequency of which cannot be estimated at present, may prove significant if attention is paid to it in future studies.

Thanks to the kindness of Dr. R. Pugh, sections have been compared with those in the collection of The Hospital for Sick Children, Great Ormond $\stackrel{\frac{0}{-}}{2}$ Street. The histological appearance of the endocardium was essentially similar, but there was no involvement of the subendocardial muscle such as was present in my material.

Two further examples of cardiac fibroelastosis $\frac{5}{\sigma}$ associated with other conditions have been seen $\stackrel{2}{2}$ at the Fountain Hospital: one with gargoylism is and the other with dermatomyositis. It would $\vec{\circ}$ appear, therefore, that fibroelastosis of the heart $\overrightarrow{-}$ cannot be regarded as a specific structural change $\vec{\omega}$ occurring in one disease only, even though there are certain cases in which it is the sole abnormality? seen at necropsy.

\section{Summary}

Structural abnormalities in epiloia are found in $\stackrel{\circ}{\rightarrow}$ the brain, skin, heart, kidney, lungs, bones and, $\vec{z}$ occasionally, other organs. The common lesions are tuberous sclerosis of the brain, adenoma $\overrightarrow{0}$ sebaceum of the face, purkinjeoma (rhabdomyoma) of of the heart, renal tumours, and phakoma of the retina. Incomplete forms of the disease are also seen, particularly among the relatives of the patients. Two cases of fibroelastosis of the heart $\bar{O}$ were encountered in the present series, and another $\frac{O}{D}$ two cases had been previously recorded in the $\stackrel{\Phi}{2}$ literature. This association may prove to be more $\overrightarrow{\vec{\circ}}$ frequent if attention is paid to it in future studies. 3 Fibroelastosis has also been seen in association with other diseases and cannot therefore be ? regarded as a specific change occurring in only흥 one disease.

\section{REFERENCES}

Critchley, M., and Earl, C. J. C. (1932). Brain, 55, 311. Gowing, N. F. C. (1953). J. Path. Bact., 65, 13. Norman, R. M., and Taylor, A. L. (1940). Ibid, 50, 61. 